\title{
Tip-enhanced Raman spectroscopy of confined carbon chains
}

\section{Review Article}

Author(s):

Tschannen, Cla Duri; Vasconcelos, Thiago L.; Novotny, Lukas (i)

Publication date:

2022-01-28

Permanent link:

https://doi.org/10.3929/ethz-b-000532084

Rights / license:

In Copyright - Non-Commercial Use Permitted

Originally published in:

The Journal of Chemical Physics 156(4), https://doi.org/10.1063/5.0073950

Funding acknowledgement:

192362 - Antenna-coupled Optoelectronics (SNF) 


\section{GHzürich}

\section{Research Collection}

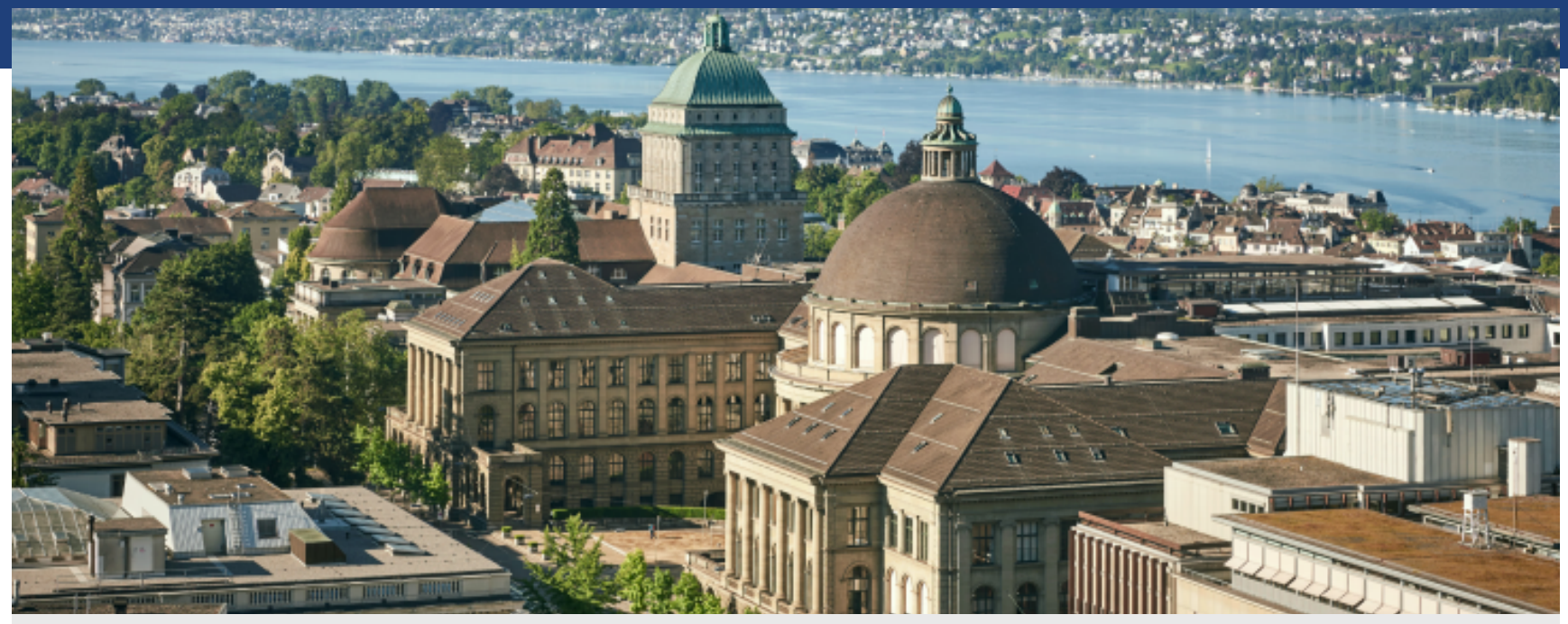

Review Article

\section{Tip-enhanced Raman spectroscopy of confined carbon chains}

Author(s):

Tschannen C.D.; Vasconcelos T.L.; Novotny L.

Publication Date:

2022-01-28

\section{Originally published in:}

Journal of Chemical Physics 156(4), http://doi.org/10.1063/5.0073950 $\rightarrow$

Rights / License:

In Copyright - Non-Commercial Use Permitted $\rightarrow$ 


\section{Tip-enhanced Raman spectroscopy of confined carbon chains}

Cite as: J. Chem. Phys. 156, 044203 (2022); https://doi.org/10.1063/5.0073950

Submitted: 05 October 2021 - Accepted: 30 December 2021 - Accepted Manuscript Online: 03 January 2022 - Published Online: 25 January 2022

(iD) Cla Duri Tschannen, (D) Thiago L. Vasconcelos and (iD) Lukas Novotny

\section{COLLECTIONS}

Paper published as part of the special topic on The Ever-Expanding Optics of Single-Molecules and Nanoparticles
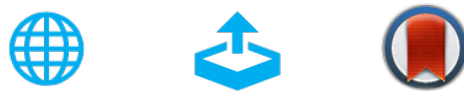

View Online

Export Citation

\section{ARTICLES YOU MAY BE INTERESTED IN}

Excitation localization in a trimeric perylenediimide macrocycle: Synthesis, theory, and single molecule spectroscopy

The Journal of Chemical Physics 156, 044304 (2022); https://doi.org/10.1063/5.0077676

Two-atomic-layered optoelectronic device enabled by charge separation on graphene/ semiconductor interface

The Journal of Chemical Physics 156, 044704 (2022); https://doi.org/10.1063/5.0065110

Cavity-enhanced saturated absorption spectroscopy of the (30012) - (00001) band of ${ }^{12} \mathrm{C}^{16} \mathrm{O}_{2}$

The Journal of Chemical Physics 156, 044201 (2022); https://doi.org/10.1063/5.0074713

\section{Chemical Physics Reviews}

\section{First Articles Now Online! $=$ READ NOW »»}




\title{
Tip-enhanced Raman spectroscopy of confined carbon chains
}

\author{
Cite as: J. Chem. Phys. 156, 044203 (2022); doi: 10.1063/5.0073950 \\ Submitted: 5 October 2021 • Accepted: 30 December 2021 • \\ Published Online: 25 January 2022
}

\section{Cla Duri Tschannen, ${ }^{1, a)}$ (D) Thiago L. Vasconcelos, ${ }^{2}$ (D) and Lukas Novotny}

\author{
AFFILIATIONS \\ ${ }^{1}$ Photonics Laboratory, ETH Zürich, 8093 Zürich, Switzerland \\ ${ }^{2}$ Materials Metrology Division, Instituto Nacional de Metrologia Qualidade e Tecnologia (INMETRO), \\ 25250-020 Duque de Caxias, RJ, Brazil
}

\begin{abstract}
Note: This paper is part of the JCP Special Topic on the Ever-Expanding Optics of Single-Molecules and Nanoparticles. a) Author to whom correspondence should be addressed: clat@ethz.ch
\end{abstract}

\begin{abstract}
Long linear chains of carbon encapsulated in carbon nanotubes represent the finite realization of carbyne, the truly one-dimensional carbon allotrope. Driven by advances in the synthesis of such structures, carbyne has attracted significant interest in recent years, with numerous experimental studies exploring its remarkable properties. As for other carbon nanomaterials, Raman spectroscopy has played an important role in the characterization of carbyne. In particular, tip-enhanced Raman scattering (TERS) has enabled imaging and spectroscopy down to the single-chain level. In this article, we provide a general introduction to carbyne and discuss the principles and experimental implementation of TERS as a key technology for the investigation of this material system. Within this context, the development of optical nanoantennas as TERS probes is addressed. We then summarize the latest progress in the Raman spectroscopic characterization of confined carbyne, with a focus on the findings assisted by TERS. Finally, we discuss open questions in the field and outline how TERS can contribute to solving them in future studies.
\end{abstract}

Published under an exclusive license by AIP Publishing. https://doi.org/10.1063/5.0073950

\section{INTRODUCTION}

Carbyne consists of an infinite chain of sp-hybridized carbon atoms and represents the truly one-dimensional allotrope of carbon. ${ }^{1,2}$ Carbyne can exist in two different structural forms commonly characterized by the bond length alternation (BLA), which is the bond length difference between adjacent bonds. For zero BLA, carbyne assumes a cumulenic structure with equidistantly arranged carbon atoms connected by double bonds. In the case of a finite BLA, the structure of carbyne is polyynic with alternating single and triple bonds. ${ }^{3,4}$ According to Peierls's theorem, the polyynic structure is energetically favorable. ${ }^{5}$ As a consequence, the equilibrium structure of carbyne is characterized by a biatomic unit cell. This dimerization opens up a direct bandgap and is the origin of the singular Raman active mode of carbyne, termed $\mathrm{C}$-mode. The $\mathrm{C}$-mode is a longitudinal optical phonon close to the $\Gamma$ point and corresponds to an in-phase stretching of the triple bonds along the chain..$^{6-8}$ The energy of the bandgap and the frequency of the $\mathrm{C}$-mode are linearly related and both depend on the extent of the BLA.

The synthesis of carbyne was long restricted to very short linear carbon chains with a maximum length of 44 atoms due to the high chemical instability of the unsaturated bonds. ${ }^{10,11}$ The BLA of such short chains, and therefore their electronic and vibrational properties, is modified by the chain length (i.e., number of carbon atoms) and by the end-capping groups. ${ }^{3,4,12,13}$ A major leap forward has been achieved by growing carbon chains inside carbon nanotubes, which act as nanoreactors enabling the growth of chains with lengths up to several thousand atoms and as protective nanocontainers shielding the chains from interaction with the environment. ${ }^{14-23}$ The properties of these long carbon chains do not exhibit any length-dependence, indicating that they have converged to the limit of infinite length. Linear carbon chains beyond $\sim 100$ atoms $^{1,7}$ can therefore be considered as a finite realization of carbyne.

Theoretical studies have stirred significant interest in carbyne by predicting outstanding mechanical, ${ }^{24}$ thermal, ${ }^{25}$ and electronic $^{26}$ properties. Experimentally, carbyne was first resolved by high resolution transmission electron microscopy, ${ }^{14,27}$ but with limited analytical information. A much more prominent role in the investigation of carbyne has been played by Raman spectroscopy. In particular, tip-enhanced Raman scattering (TERS) has enabled spectroscopic imaging of single carbyne chains with nanometric 
resolution. In this work, we provide an overview of the recent advances in the characterization of carbyne chains confined inside carbon nanotubes, including our own contributions. We first review the conceptual and experimental principles of TERS and discuss the progress made in the development of optical nanoantennas as TERS probes in recent years. Then, we summarize the most important findings assisted by TERS measurements of confined carbyne, including the interaction with the encasing host nanotube and the quantification of carbyne's Raman response. Finally, we outline some open questions to be addressed in future studies of confined carbyne.

\section{TIP-ENHANCED RAMAN SCATTERING}

\section{A. Principles}

Raman spectroscopy is based on the inelastic scattering of monochromatic light by vibrational modes in molecules or materials. ${ }^{28}$ In a Raman spectrum, the frequency of a vibrational mode is determined by the energy difference between incoming and outgoig photons, and reflects the bond strength between and masses of the constituent atoms. ${ }^{29,30}$ However, as in any conventional optical microscopy technique, the spatial resolution of Raman spectroscopy is fundamentally limited by diffraction to roughly half the wavelength of the incident light. ${ }^{31,32}$ The smallest achievable resolution when using visible light is therefore on the order of a few hundred nanometers, which is insufficient for resolving and characterizing the morphology of carbyne or any other nanostructure.

The diffraction limit arises from the fact that the high spatial frequency components of optical fields are lost upon propagation, that is, they form evanescent waves that decay exponentially away from the source. Hence, there is always a loss of information from the near field to the far field. ${ }^{33}$ Improving the resolution beyond the diffraction limit thus requires either prior information or the detection of near-field information (i.e., evanescent waves) in the far field. In TERS, this is achieved by placing a sharp metal tip sufficiently close to the sample to scatter the near-field components into propagating fields that can be detected in the far field. ${ }^{34,35}$ However, this also implies that in the far field, near-field and far-field components are intermixed. Therefore, in order to achieve sub-diffraction resolution, the near-field signal needs to be enhanced to be the dominant signal contribution. The second purpose of a TERS tip is thus to locally enhance the incoming and scattered radiation. This leads to an enhancement of Raman scattering, which scales roughly with the fourth power of the electric field enhancement provided by the tip. ${ }^{33}$ The overall signal enhancement, defined as the ratio of the Raman signal recorded with and without the tip, depends on the sample size and the symmetry of the interrogated vibrational mode. $^{36-38}$

In summary, the task performed by a TERS tip is that of an optical nanoantenna, namely, localizing incoming radiation to a subwavelength volume and converting evanescent field components into propagating waves that can be detected in the far field. By interconverting and enhancing propagating radiation and local near fields, nanoantennas have enabled Raman spectroscopy to be performed with spatial resolution down to $10 \mathrm{~nm} .{ }^{39}$

\section{B. Experimental implementation}

Our TERS setup is similar to that described in Refs. 34 and 40 and schematically illustrated in Fig. 1. It consists of a home-built atomic force microscope (AFM) scan head designed for non-contact shear-force operation with quartz tuning forks, on top of an inverted confocal microscope equipped with an $x, y$-scan stage for rasterscanning a transparent sample sitting on a thin glass coverslip. The exciting HeNe laser beam $(\lambda=632.8 \mathrm{~nm})$ is converted from linear to radial polarization (doughnut mode) to ensure a strong longitudinal electric field component in the focus, which is required to excite a locally enhanced and confined field at the nanoantenna apex. ${ }^{33,41}$ The laser beam is then reflected by a dichroic beam splitter and strongly focused onto the sample surface from below through an oil immersion high numerical aperture (NA 1.4) objective lens. From

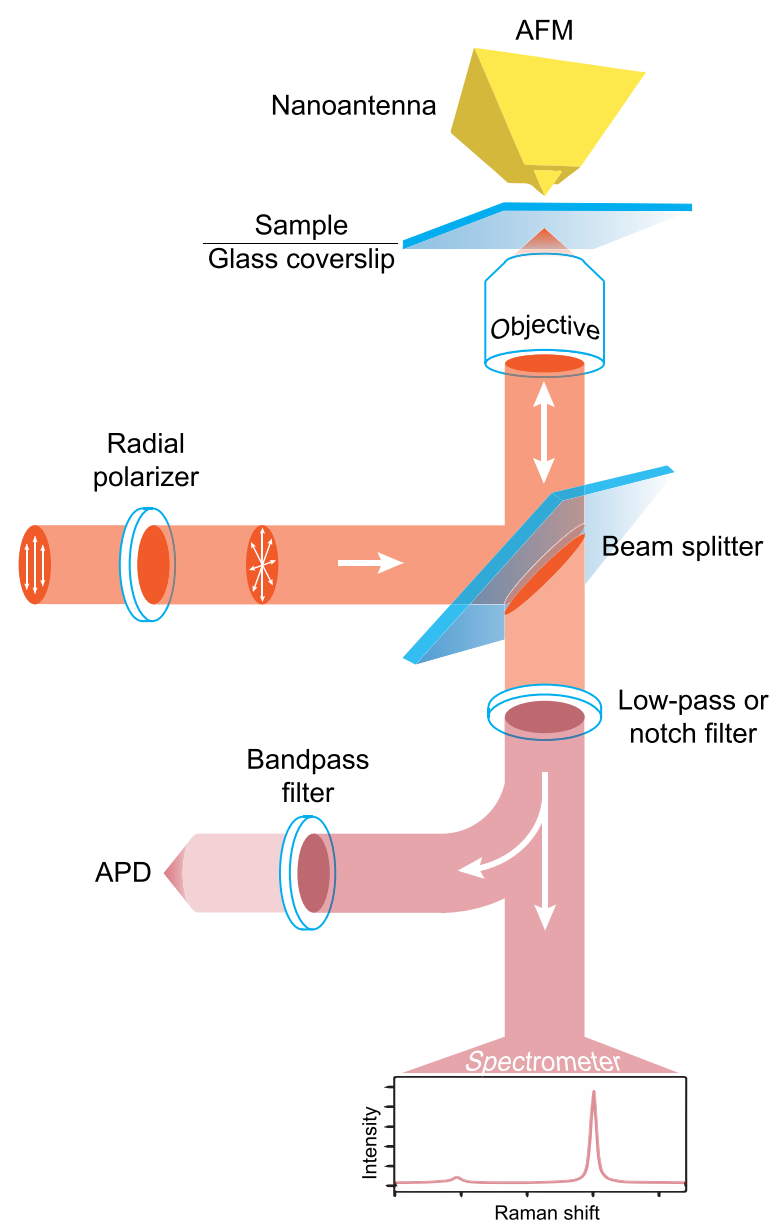

FIG. 1. Schematic illustration of the TERS setup. A radially polarized HeNe laser beam is strongly focused onto the sample surface by a high NA objective, generating longitudinal electric field components in the focus. Positioning an optical nanoantenna into the focus generates a localized excitation source for Raman scattering. The tip-sample distance is controlled by an atomic force microscope (AFM) scan head. The backscattered light is collected by the same objective, filtered by appropriate interference filters, and guided by a flip-mirror to either an avalanche photodiode (APD) or a CCD-equipped spectrometer. 
the other side of the substrate, a nanoantenna, attached to the end of a quartz tuning fork, is positioned into the center of the laser focus and held at a distance of $\sim 5 \mathrm{~nm}$ from the sample surface by means of the shear-force feedback. The scattered light is collected by the same objective, passed through the beam splitter, and filtered by a low pass or notch filter to remove the Rayleigh component. Finally, the Raman scattered light is either detected by a CCD-equipped spectrometer or collected by an avalanche photodiode (APD) after the spectral region of interest is selected by an appropriate optical bandpass filter. Optical and topographic images are collected simultaneously by raster-scanning. ${ }^{42}$

\section{Development of optical antennas for TERS}

In a TERS system as described above, the optical nanoantenna constitutes the key element for determining the achievable spatial resolution as well as the signal enhancement. ${ }^{41,43}$ The spatial resolution is defined by the nanoantenna apex diameter, usually around $20 \mathrm{~nm}$. The signal enhancement, on the other hand, is driven by the optical efficiency of the nanoantenna, that is, its capability to harness near-field optical information by locally enhancing the electric fields. In general, the nanoantenna's efficiency is ruled by two main field enhancement mechanisms, one being of purely geometric nature, while the other relies on a plasmonic resonance effect. ${ }^{41}$ The geometric contribution originates from the lightning rod effect (LRE) associated with the high curvature at the tip end. ${ }^{44,45}$ For instance, the field enhancement provided by semi-infinite tapered gold tips relies purely on the LRE and is typically rather moderate. This applies to electrochemically etched gold wires [see Fig. 2(a)], which have long been the most common probes for shear-force AFM-based TERS. ${ }^{46}$ Besides the low optical efficiency, other drawbacks of etched wires are that their fabrication offers low yield and low reproducibility of the tip morphology. ${ }^{47}$ A remarkable improvement in this regard has been achieved by the template-stripping fabrication technique demonstrated by Johnson et al. in $2012 .{ }^{48}$ This method is based on the use of lithography to introduce micropyramidal cavities in a silicon substrate, followed by deposition of a thin gold film onto the template. In this way, more than a million identical probes can be fabricated over a wafer in parallel, each of which can be stripped on-demand, one at a time [see Fig. 2(b)]. Moreover, the micropyramid's large open semi-angle $\left(35.2^{\circ}\right)$ in combination with the small apex size $(\sim 20 \mathrm{~nm})$ was shown to optimize the LRE signal enhancement mechanism. ${ }^{48,49}$

Nanoantenna geometries that exploit localized surface plasmon resonances (LSPRs) are able to provide stronger signal enhancements than those relying solely on the LRE. ${ }^{41}$ Such resonances are supported by plasmonic nanostructures of subwavelength dimension, where the spatial confinement on the surface gives rise to spectrally discrete dipolar or monopolar modes. ${ }^{51,52}$ Upon excitation with incident radiation that is resonant with one of these modes, strong local field enhancement is observed in the vicinity of the nanostructure. Based on this idea, numerous studies were published
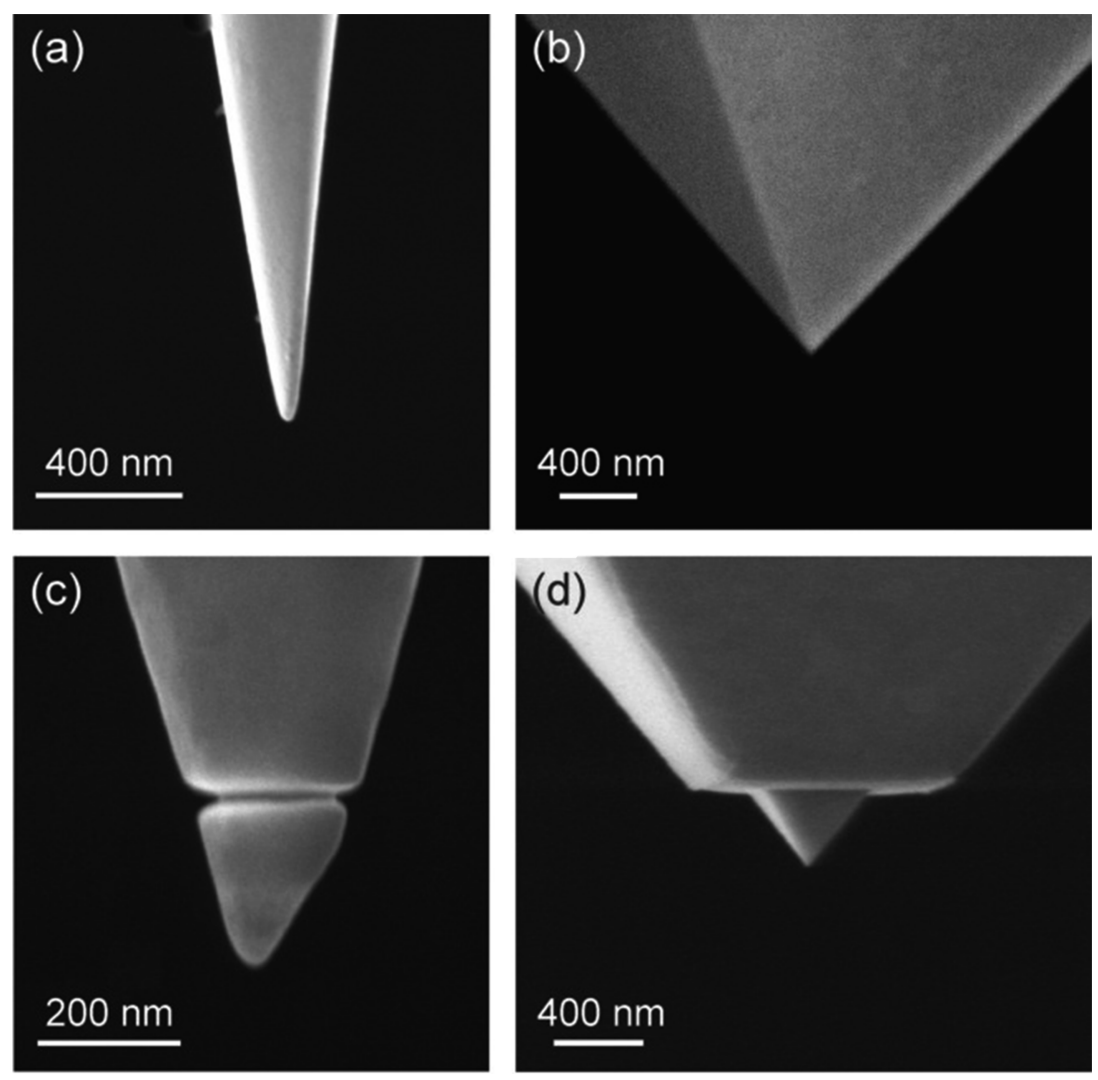

FIG. 2. Scanning electron microscopy images of different TERS nanoantenna geometries. (a) Electrochemically etched gold wire. (b) Template-stripped gold micropyramid. (c) Etched gold wire as shown in (a) but with a superficial line groove created by focused ion beam milling. The groove is located $240 \mathrm{~nm}$ from the tip apex such that the first dipolar mode supported by the structure is resonant with the HeNe laser line. Adapted with permission from Vasconcelos et al., ACS Nano 9, 6297 (2015). Copyright 2015 American Chemical Society. (d) Plasmon-tunable tip pyramid (PTTP) nanoantenna with a nanopyramid edge size of $470 \mathrm{~nm}$, tuned to be in resonance with the second monopole mode at the HeNe laser line. 
using gold or silver nanoparticles supporting LSPR modes at the laser wavelength as optical antennas for tip-enhanced spectroscopy, demonstrating the importance of plasmonic resonances for achieving large signal enhancements. ${ }^{53-57}$ In a different approach, the authors of Ref. 50 showed that a groove along the circumference of a tapered gold tip [see Fig. 2(c)] is sufficient to generate LSPR modes. By setting the distance between the line groove and the tip apex such that the first supported electric dipole mode is resonant with the excitation wavelength, the signal enhancement was improved 5.5 times. The same concept was applied by Maouli et al. to fabricate silver-coated $\mathrm{SiO}_{2}$-based antennas with a mushroom-shaped apex that supports LSPR modes. ${ }^{58}$

Recently, a new fabrication method was introduced for template-stripped nanoantennas that combines the high yield and reproducibility of the gold micropyramids with the strong field enhancement afforded by LSPR. ${ }^{59}$ The technique is based on a two-step lithography process to generate gold structures consisting of a micropyramidal frustum with a nanopyramidal end [see Fig. 2(d)]. The size of the nanopyramid can be adjusted to tune the second LSPR electric monopole mode into resonance with the laser wavelength. The resulting nanoantenna geometry has therefore been termed plasmon-tunable tip pyramid (PTTP). By combining the LRE-optimizing pyramidal shape with a morphological barrier capable of supporting the LSPR mode that leads to the highest field intensity, ${ }^{60}$ the PTTP probes have enabled some of the largest signal enhancements reported in the literature. ${ }^{41,61,62}$ In addition, the PTTP fabrication method was shown to further improve the reproducibility, producing nanoantennas with an apex diameter smaller than $30 \mathrm{~nm}$ in $92.4 \%$ of the batch. This result constitutes a substantial improvement compared to the $44.7 \%$ achieved in the fabrication of template-stripped micropyramids.

In sum, the main criteria for a practical TERS nanoantenna are spatial resolution, optical efficiency, reproducibility, and yield. The PTTP represents the most elaborate probe geometry developed to date, for it elegantly addresses all these requirements at once. For this reason, we have used PTTP probes with a plasmon resonance tuned to the wavelength of our HeNe laser for all the TERS measurements presented in this work. A representative set of TERS images of confined carbyne chains is shown in Fig. 3, evidencing the nanoscale spatial resolution afforded by TERS. The corresponding TERS spectra are plotted in Fig. 4 along with the same measurements recorded without the PTTP, underlining the strong signal enhancement provided by this TERS probe geometry.

\section{Alternative TERS configurations}

In this review, the focus lies exclusively on tuning fork-based TERS, which is the only TERS technique that has been used to date to characterize confined carbyne. However, there exist several alternative experimental implementations of TERS, both with respect to the configuration of illumination and signal collection as well as the type of feedback that is used for controlling the tip-sample distance. Some of these alternative TERS techniques utilize different kinds of tips as scanning probes than the geometries that were elaborated on in Sec. II C. Nonetheless, the mentioned main criteria for practicability-spatial resolution, optical efficiency, reproducibility, and yield-apply to TERS tips in general. For information about other experimental TERS setups and associated probes, we refer the reader to existing excellent reviews and references therein. ${ }^{64-68}$ In these articles, some more advanced topics that are not touched upon here are also covered, such as the operation of TERS in liquids or in ultrahigh vacuum, optomechanical coupling between the tip and the sample, or tip-assisted mechanical nanomanipulation.

\section{NEAR-FIELD AND FAR-FIELD RAMAN STUDIES OF CONFINED CARBYNE}

Raman spectroscopy is a versatile and non-destructive characterization method offering high chemical specificity by providing vibrational information about the molecule or material under study. In this way, Raman spectroscopy has been used in many studies to confirm the presence of confined carbyne (or short linear carbon chains) in bulk samples of carbon nanotubes, with C-mode peaks found to appear in the range between 1790 and $1860 \mathrm{~cm}^{-1}$. ${ }^{14-23}$ However, the information accessible by Raman spectroscopy is not limited to vibrational properties. For instance, resonant enhancement of Raman scattering occurs for excitation energies close to or coinciding with an optical transition. Therefore, by recording the Raman signal as a function of excitation energy, one can obtain information about the electronic structure. Shi et al. have recorded such Raman resonance profiles of six C-mode frequency bands in the range between 1793 and $1856 \mathrm{~cm}^{-1}$ from bulk quantities of carbyne chains encapsulated inside double-walled carbon nanotubes (DWCNTs) and have shown that each Raman peak can be associated with a different bandgap. ${ }^{67}$ Moreover, it was confirmed that the $\mathrm{C}$-mode frequency and bandgap energy of confined carbyne are linearly related. Hence, the information on the bandgap energy of a confined carbyne chain is contained in the center frequency of the C-mode peak in the Raman spectrum.

In a theoretical study by Wanko et al., it has been put forward that the BLA, and thus the Raman response, of confined carbyne is affected by the interaction with the encapsulating host nanotube. ${ }^{68}$ In the work of Heeg et al., this interaction has been investigated experimentally using TERS. ${ }^{69}$ They have identified 14 pairs of DWCNTs and encapsulated carbyne chains and established a correlation between their respective Raman signature. They found a linear scaling of the C-mode frequency with the diameter of the encasing inner nanotube, pointing toward van-der-Waals forces as the dominating interaction between the tube and the encapsulated chain. Importantly, such findings would not have been possible with farfield Raman measurements because they lack the spatial resolution required to ensure the presence of a single carbyne chain and to verify that the Raman signal of both chain and tube arises from the same pair. $^{70}$

Let us here briefly clarify the notion of a single carbyne chain. The characteristic feature of what is, in practice, commonly considered a single carbyne chain is a spectral fingerprint comprising a single C-mode peak (see Fig. 4), that is, a Raman peak with a line shape that is well described by a single Lorentzian. ${ }^{20,69}$ This spectral signature should be consistent along the chain, which can only be verified by TERS. A sudden change in peak position indicates the presence of another chain fragment encapsulated in a different nanotube within the same bundle. These individual contributions cannot be disentangled with far-field measurements alone. We further point out that TERS only enables estimating the chain length 

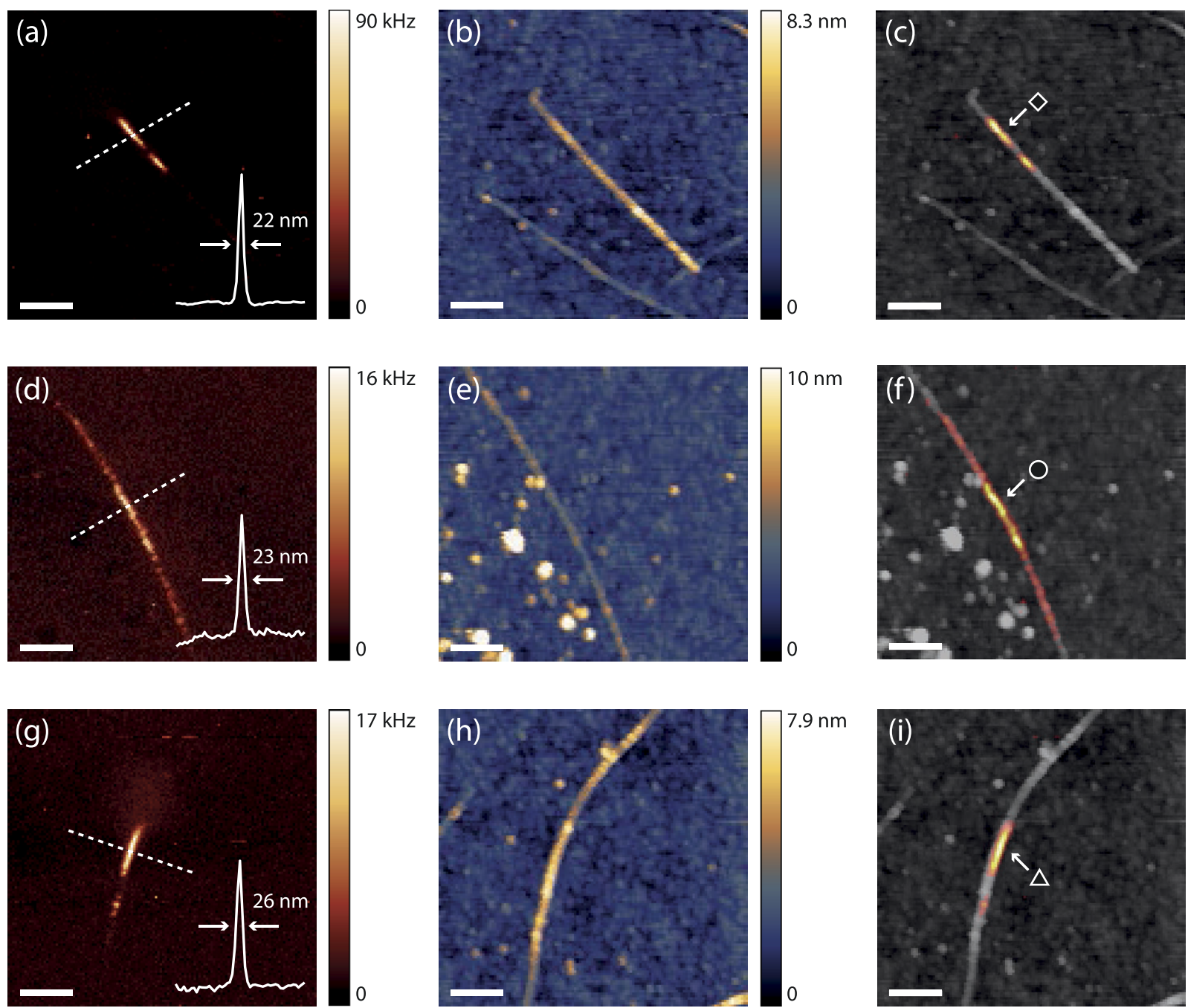

FIG. 3. TERS imaging of confined carbyne. Simultaneously acquired optical (a), (d), and (g) and topographic (b), (e), and (h) images. The insets in (a), (d), and (g) show the intensity profile extracted from the white dashed lines. The spatial resolution is indicated for each measurement. (c), (f), and (i) Superposition of optical and topographic information. TERS spectra recorded at the marked positions are shown in Fig. 4. The length of the scale bars corresponds to $200 \mathrm{~nm}$. All carbyne chains shown were fabricated as part of the same sample following the procedure of Shi et al..$^{20}$ and dispersed on a thin glass coverslip according to the recipe described in Ref. 63.

to within its resolution of around $20 \mathrm{~nm}$ and it does not permit any statement about the continuity of the chain below this limit. However, the presence of a single C-mode peak indicates that potential individual fragments are longer than $\sim 100$ atoms and have thus converged to the limit of infinite length in terms of their properties. Indeed, no dependence of the chains' properties on the length as measured by TERS was found by Heeg et al., thus corroborating that the investigated confined carbon chains are, in fact, the finite realization of carbyne.

In another study by Heeg et al., it was shown that not only the Raman peak position and the bandgap energy but also the excited state dynamics of confined carbyne critically depend on the confinement inside the host nanotube. ${ }^{63}$ The lifetime of the excited electronic state was inferred from the linewidth of a Raman resonance profile of an individual carbyne chain, which was previously identified using TERS. The authors showed that the excited state lifetime does not correlate with the bandgap energy and C-mode frequency and is therefore not directly dependent on the BLA of the carbyne chain. Rather, it is the electronic structure of the encasing nanotube, determined by its chirality, which modulates the efficiency of the energy transfer and thus the relaxation dynamics of the chain's excited electronic state.

In the same work, the polarization dependence of Raman scattering from confined carbyne was studied. It was demonstrated that the Raman signal of confined carbyne only arises from the projection of incoming and scattered light onto the chain's main 


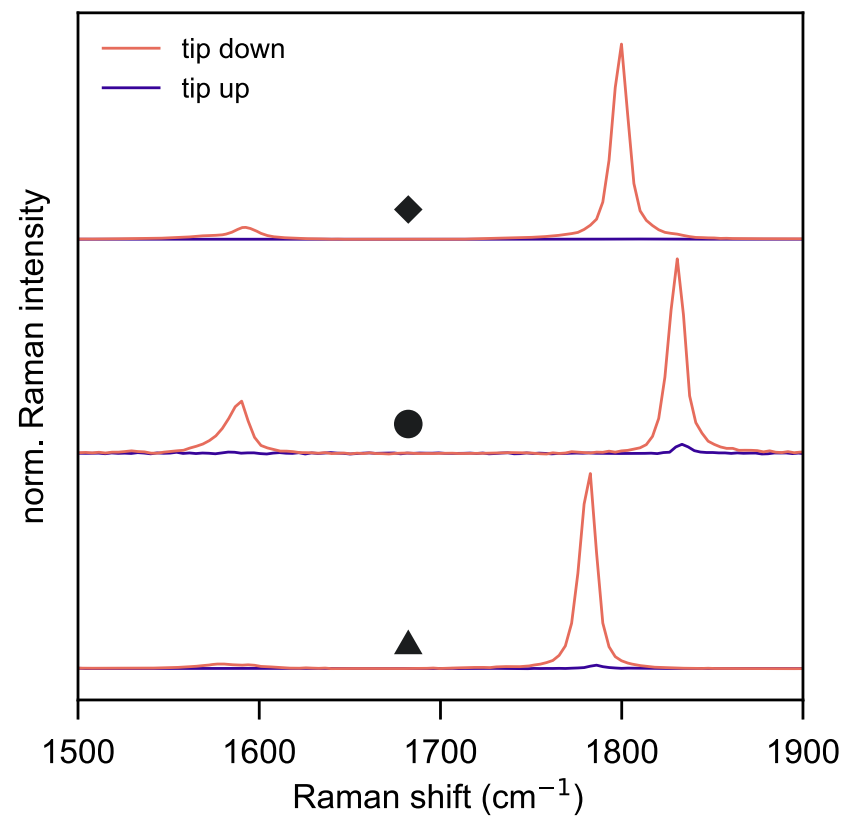

FIG. 4. TERS spectra (red are with PTTP, blue are without PTTP), of confined carbyne, recorded at the locations indicated in Fig. 3. The strong peaks around $1800 \mathrm{~cm}^{-1}$ come from the C-mode of the confined carbyne chain, whereas the signature below $1600 \mathrm{~cm}^{-1}$ arises from the encapsulating nanotube structure. The blue spectra (tip up) are far-field measurements recorded without the TERS probe but otherwise identical experimental conditions. All spectra are normalized to the C-peak height of the TERS measurements and vertically offset for better visibility. The enhancement of the C-peak area by the tip amounts to 70 (bottom), 25 (middle), and 290 (top). The different C-mode frequencies are associated with distinct structural conformations of carbyne chains encapsulated by host nanotubes of varying diameter and report different bandgap energies, as explained in the main text. A single Raman spectrum of a confined carbyne chain thus provides information about its vibrational, structural, and electronic properties.

axis. Such behavior is well known from other one-dimensional systems, such as carbon nanotubes, and originates from the anisotropic polarizability. ${ }^{71,72}$ This pronounced polarization dependence needs to be taken into account if one is to infer quantitative information about the Raman intensities of individual carbyne chains in far-field measurements.

Combined, the TERS studies by Heeg and co-workers ${ }^{63,69}$ have demonstrated that nanotubes with similar diameter will host carbyne chains with similar C-mode frequencies and bandgap energies, while the excited state lifetime may vary depending on the chirality. Therefore, the properties of confined carbyne are tunable by the choice of nanotube host. Moreover, other works have shown that this tunability also extends to external perturbations, such as pressure $^{73}$ or charge transfer. ${ }^{68,74}$

Looking at the many Raman spectra of bulk samples of carbyne confined inside carbon nanotubes available in the literature, ${ }^{14-23}$ it is apparent that the Raman response of carbyne is remarkably strong. This observation is even more striking in Raman spectra of single confined carbyne chains, ${ }^{63,69}$ where the intensity of the C-mode peak easily surpasses the G-mode signature of the encasing nanotube structure. Recently, a number has been put on this qualitative insight by rigorous quantification of the Raman scattering from confined carbyne. ${ }^{75}$ It was shown that the resonant differential Raman scattering cross section of confined carbyne is on the order of $10^{-22} \mathrm{~cm}^{2} \mathrm{sr}^{-1}$ per atom, exceeding that of any other known material or molecule by 2 orders of magnitude or more. The use of TERS was crucial in this study to locate 20 individual confined carbyne chains and measure their length for later signal normalization.

Its strong Raman response renders carbyne a promising candidate for experiments that rely on strong interaction between light and vibrational modes or applications such as biological imaging. Another possible application, which was recently investigated, is nanoscale thermometry. ${ }^{62}$ TERS was used to locate an individual confined carbyne chain, which was subsequently heated by resonant excitation with varying laser powers. Based on the anti-Stokes/Stokes ratio the laser-induced heating was quantified, thus demonstrating the potential of carbyne for local temperature monitoring.

\section{OPEN QUESTIONS}

Although TERS has assisted numerous findings that have advanced our understanding of confined carbyne, there are still many aspects that have remained unexplored to date. For instance, the intrinsic phonon lifetime of the $\mathrm{C}$-mode, reflected by its fullwidth at half-maximum (FWHM), has not been systematically examined. In Fig. 5(a), we plot the FWHM of 48 individual carbyne chains. Each FWHM value was obtained from a TERS spectrum by first removing a linear background and then fitting a Lorentzian line shape to the data, as illustrated in Fig. 5(b). The mean FHWM of these measurements is $11.1 \mathrm{~cm}^{-1}$, with the smallest and largest value amounting to 8.2 and $16.7 \mathrm{~cm}^{-1}$, respectively. This large betweenchain heterogeneity emphasizes the need for studying the phonon dynamics of confined carbyne at the single-chain level. While longitudinal optical phonons, such as the C-mode, typically decay via a three-phonon process into two acoustic modes, ${ }^{76}$ the temperature dependence of the C-mode FWHM observed in bulk measurements suggests an additional decay channel through vibrational coupling to the radial-breathing mode (RBM) of the host nanotube. ${ }^{18}$ Thus, temperature-dependent Raman measurements of individual carbyne chains are required to unravel the intrinsic phonon lifetime of the C-mode and its decay dynamics as well as the potential influence thereon of the host nanotube properties. Because scattering by optical phonons provides a channel for charge carrier relaxation, the characterization of the $\mathrm{C}$-mode phonon dynamics will provide novel insights into the transport properties of confined carbyne. $^{77,78}$

Another important property of the C-mode, which has not yet been experimentally investigated, is its spatial coherence length. The phonon coherence length is accessible in TERS experiments via the distance dependence of the tip-induced signal enhancement. ${ }^{37,79}$ Knowledge about the spatial phonon coherence of the C-mode will not only push our understanding of the thermal properties of confined carbyne ${ }^{80,81}$ but is required for a quantitative understanding of the tip-sample interaction and associated scattering enhancement in TERS experiments of confined carbyne. ${ }^{38}$

Finally, we see a vast playground for future TERS studies on the anti-Stokes side of carbyne's Raman spectrum. The combination of carbyne's record-high Raman scattering cross section and strong 
(a)

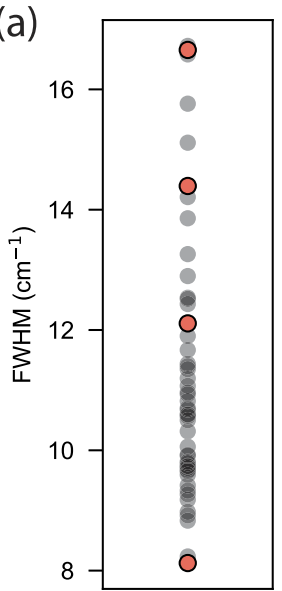

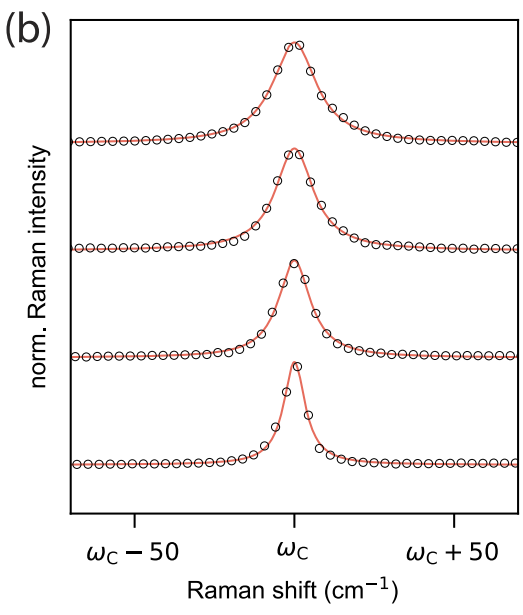

FIG. 5. Full-width at half-maximum (FWHM) of confined carbyne. (a) Distribution of FWHM values obtained from TERS spectra of 48 different confined carbyne chains. (b) Spectra (black circles) and Lorentzian fits (red lines) of the data points highlighted in (a). The spectra are centered around the respective C-mode frequency $\omega_{\mathrm{C}}$ to facilitate the comparison, normalized to the height of the C-peak, and vertically offset for better visibility.

field enhancement provided by the PTTP TERS probes could possibly lead to interesting phenomena, such as vibrational pumping, as demonstrated for dye molecules under surface-enhanced Raman scattering (SERS) conditions. ${ }^{82}$ Vibrational pumping is characterized by a significant contribution of phonons induced by a Stokes scattering process to the overall phonon population, which leads to a quadratic dependence of the anti-Stokes signal on the excitation power. Such a non-linear power dependence of the anti-Stokes process has also been observed in conventional Raman spectroscopy of twisted-bilayer graphene (tBLG) tuned to work in resonance with the anti-Stokes emission. ${ }^{83}$ Hence, by using chains with a bandgap energy close to the energy of C-mode scattered anti-Stokes photons, the presumed strong anti-Stokes TERS signal of confined carbyne can possibly be further increased.

\section{CONCLUSION}

Raman spectroscopy, in particular TERS, has proved to be an invaluable tool to study the properties of confined carbyne. By providing the spatial resolution necessary to ensure that only a single carbyne chain is spectroscopically addressed, TERS has enabled studies on individual carbyne chains, which have unraveled intrinsic properties and inter-chain heterogeneities that are masked in far-field measurements of bulk samples. In this way, it was demonstrated that the interaction with the host nanotube, characterized by its chirality, is central in determining the electronic and vibrational properties of the encapsulated carbyne chain. Furthermore, single-chain studies have revealed that confined carbyne is the strongest known Raman scatterer and that individual carbyne chains can potentially be used for localized thermometry. The recent progress in the development of optical nanoantennas makes TERS measurements reproducible and efficient, which is key for the further characterization of confined carbyne. In addition, the use of alternative characterization techniques, such as absorption or photoluminescence spectroscopy, is prohibited by the carbon nanotubes due to spectral overlap or quenching, respectively. ${ }^{19,70,72,84}$ We therefore expect TERS to continue playing a leading role in the experimental investigation of confined carbyne in future studies.

\section{ACKNOWLEDGMENTS}

This work was supported by the Swiss National Science Foundation (Grant No. 200020_192362/1). T.L.V. acknowledges financial support from Finep (SibratecNano 21040). The authors acknowledge INMETRO and UFMG for the PTTP nanoantennas. Thomas Pichler and Lei Shi are gratefully acknowledged for the carbyne sample.

\section{AUTHOR DECLARATIONS \\ Conflict of Interest}

The authors have no conflicts of interest to disclose.

\section{DATA AVAILABILITY}

The data that support the findings of this study are available from the corresponding author upon reasonable request.

\section{REFERENCES}

${ }^{1}$ R. B. Heimann, S. E. Evsyukov, and L. Kavan, Carbyne and Carbynoid Structures (Springer Science \& Business Media, 1999).

${ }^{2}$ A. Hirsch, Nat. Mater. 9, 868 (2010).

${ }^{3}$ C. S. Casari, M. Tommasini, R. R. Tykwinski, and A. Milani, Nanoscale 8, 4414 (2016).

${ }^{4}$ C. S. Casari and A. Milani, MRS Commun. 8, 207 (2018).

${ }^{5}$ R. E. Peierls, Quantum Theory of Solids (Clarendon Press, Oxford, 1955).

${ }^{6}$ J. Kastner, H. Kuzmany, L. Kavan, F. P. Dousek, and J. Kuerti, Macromolecules 28, 344 (1995).

${ }^{7}$ J. Kürti and H. Kuzmany, Phys. Rev. B 44, 597 (1991).

${ }^{8}$ A. Milani, M. Tommasini, D. Fazzi, C. Castiglioni, M. D. Zoppo, and G. Zerbi, J. Raman Spectrosc. 39, 164 (2008).

${ }^{9}$ A. Milani, M. Tommasini, and G. Zerbi, J. Raman Spectrosc. 40, 1931 (2009).

${ }^{10}$ T. Gibtner, F. Hampel, J.-P. Gisselbrecht, and A. Hirsch, Chem. - Eur. J. 8, 408 (2002).

${ }^{11}$ W. A. Chalifoux and R. R. Tykwinski, Nat. Chem. 2, 967 (2010).

${ }^{12}$ M. Tommasini, A. Milani, D. Fazzi, A. Lucotti, C. Castiglioni, J. A. Januszewski, D. Wendinger, and R. R. Tykwinski, J. Phys. Chem. C 118, 26415 (2014).

${ }^{13}$ A. Milani, V. Barbieri, A. Facibeni, V. Russo, A. Li Bassi, A. Lucotti, M. Tommasini, M. D. Tzirakis, F. Diederich, and C. S. Casari, Sci. Rep. 9, 1648 (2019).

${ }^{14}$ X. Zhao, Y. Ando, Y. Liu, M. Jinno, and T. Suzuki, Phys. Rev. Lett. 90, 187401 (2003).

${ }^{15}$ M. Endo, Y. A. Kim, T. Hayashi, H. Muramatsu, M. Terrones, R. Saito, F. Villalpando-Paez, S. G. Chou, and M. S. Dresselhaus, Small 2, 1031 (2006).

${ }^{16}$ M. Jinno, Y. Ando, S. Bandow, J. Fan, M. Yudasaka, and S. Iijima, Chem. Phys. Lett. 418, 109 (2006).

${ }^{17}$ C. Fantini, E. Cruz, A. Jorio, M. Terrones, H. Terrones, G. Van Lier, J.-C. Charlier, M. S. Dresselhaus, R. Saito, Y. A. Kim, T. Hayashi, H. Muramatsu, M. Endo, and M. A. Pimenta, Phys. Rev. B 73, 193408 (2006).

${ }^{18}$ L. Shi, L. Sheng, L. Yu, K. An, Y. Ando, and X. Zhao, Nano Res. 4, 759 (2011).

${ }^{19}$ N. F. Andrade, T. L. Vasconcelos, C. P. Gouvea, B. S. Archanjo, C. A. Achete, Y. A. Kim, M. Endo, C. Fantini, M. S. Dresselhaus, and A. G. Souza Filho, Carbon 90, 172 (2015). 
${ }^{20}$ L. Shi, P. Rohringer, K. Suenaga, Y. Niimi, J. Kotakoski, J. C. Meyer, H. Peterlik, M. Wanko, S. Cahangirov, A. Rubio, Z. J. Lapin, L. Novotny, P. Ayala, and T. Pichler, Nat. Mater. 15, 634 (2016).

${ }^{21}$ Y. Zhang, J. Zhao, Y. Fang, Y. Liu, and X. Zhao, Nanoscale 10, 17824 (2018).

${ }^{22}$ S. Toma, K. Asaka, M. Irita, and Y. Saito, Surf. Interface Anal. 51, 131 (2019).

${ }^{23}$ L. Shi, R. Senga, K. Suenaga, H. Kataura, T. Saito, A. P. Paz, A. Rubio, P. Ayala, and T. Pichler, Nano Lett. 21, 1096 (2021).

${ }^{24}$ M. Liu, V. I. Artyukhov, H. Lee, F. Xu, and B. I. Yakobson, ACS Nano 7, 10075 (2013).

${ }^{25} \mathrm{M}$. Wang and S. Lin, Sci. Rep. 5, 18122 (2015).

${ }^{26}$ S. Tongay, R. T. Senger, S. Dag, and S. Ciraci, Phys. Rev. Lett. 93, 136404 (2004).

${ }^{27}$ Z. Wang, X. Ke, Z. Zhu, F. Zhang, M. Ruan, and J. Yang, Phys. Rev. B 61, R2472 (2000).

${ }^{28}$ C. V. Raman and K. S. Krishnan, Nature 121, 501 (1928).

${ }^{29}$ R. L. McCreery, Raman Spectroscopy for Chemical Analysis (John Wiley \& Sons, 2000).

${ }^{30}$ A. Jorio, M. S. Dresselhaus, R. Saito, and G. Dresselhaus, Raman Spectroscopy in Graphene Related Systems (John Wiley \& Sons, 2011).

${ }^{31}$ E. Abbe, Arch. Mikrosk. Anat. 9, 413 (1873).

${ }^{32}$ L. Rayleigh, London, Edinburgh Dublin Philos. Mag. J. Sci. 42, 167 (1896).

${ }^{33}$ L. Novotny and B. Hecht, Principles of Nano-Optics (Cambridge University Press, Cambridge, 2012).

${ }^{34}$ L. G. Cançado, A. Hartschuh, and L. Novotny, J. Raman Spectrosc. 40, 1420 (2009).

${ }^{35}$ R. Beams, J. Raman Spectrosc. 49, 157 (2018).

${ }^{36}$ D. Roy, J. Wang, and C. Williams, J. Appl. Phys. 105, 013530 (2009).

${ }^{37}$ R. Beams, L. G. Cançado, S.-H. Oh, A. Jorio, and L. Novotny, Phys. Rev. Lett. 113, 186101 (2014).

${ }^{38}$ L. G. Cançado, R. Beams, A. Jorio, and L. Novotny, Phys. Rev. X 4, 031054 (2014).

${ }^{39}$ L. Novotny and N. Van Hulst, Nat. Photonics 5, 83 (2011).

${ }^{40}$ Z. J. Lapin, R. Beams, L. G. Cançado, and L. Novotny, Faraday Discuss. 184, 193 (2015).

${ }^{41}$ T. L. Vasconcelos, B. S. Archanjo, B. S. Oliveira, W. F. Silva, R. S. Alencar, C. Rabelo, C. A. Achete, A. Jorio, and L. G. Cançado, IEEE J. Sel. Top. Quantum Electron. 27(1), 4600411 (2020).

${ }^{42}$ E. H. Synge, London, Edinburgh Dublin Philos. Mag. J. Sci. 13, 297 (1932).

${ }^{43}$ X. Shi, N. Coca-López, J. Janik, and A. Hartschuh, Chem. Rev. 117, 4945 (2017).

${ }^{44}$ L. Novotny, R. X. Bian, and X. S. Xie, Phys. Rev. Lett. 79, 645 (1997).

${ }^{45}$ N. Mauser and A. Hartschuh, Chem. Soc. Rev. 43, 1248 (2014).

${ }^{46}$ B. Ren, G. Picardi, and B. Pettinger, Rev. Sci. Instrum. 75, 837 (2004).

${ }^{47}$ T. Deckert-Gaudig, A. Taguchi, S. Kawata, and V. Deckert, Chem. Soc. Rev. 46, 4077 (2017).

${ }^{48}$ T. W. Johnson, Z. J. Lapin, R. Beams, N. C. Lindquist, S. G. Rodrigo, L. Novotny, and S.-H. Oh, ACS Nano 6, 9168 (2012).

${ }^{49}$ S. Thomas, G. Wachter, C. Lemell, J. Burgdörfer, and P. Hommelhoff, New J. Phys. 17, 063010 (2015)

${ }^{50}$ T. L. Vasconcelos, B. S. Archanjo, B. Fragneaud, B. S. Oliveira, J. Riikonen, C. Li, D. S. Ribeiro, C. Rabelo, W. N. Rodrigues, A. Jorio, C. A. Achete, and L. G. Cançado, ACS Nano 9, 6297 (2015).

${ }^{51}$ K. L. Kelly, E. Coronado, L. L. Zhao, and G. C. Schatz, J. Phys. Chem. B 107, 668 (2003).

${ }^{52}$ P. K. Jain, X. Huang, I. H. El-Sayed, and M. A. El-Sayed, Acc. Chem. Res. 41, 1578 (2008).

${ }^{53}$ P. Bharadwaj and L. Novotny, Opt. Express 15, 14266 (2007).

${ }^{54}$ P. Bharadwaj and L. Novotny, J. Phys. Chem. C 114, 7444 (2010).
${ }^{55}$ M. Fleischer, A. Weber-Bargioni, M. V. P. Altoe, A. M. Schwartzberg, P. J. Schuck, S. Cabrini, and D. P. Kern, ACS Nano 5, 2570 (2011).

${ }^{56}$ C. Höppener, Z. J. Lapin, P. Bharadwaj, and L. Novotny, Phys. Rev. Lett. 109, 017402 (2012).

${ }^{57}$ A. Taguchi, J. Yu, P. Verma, and S. Kawata, Nanoscale 7, 17424 (2015).

${ }^{58}$ I. Maouli, A. Taguchi, Y. Saito, S. Kawata, and P. Verma, Appl. Phys. Express 8, 032401 (2015).

${ }^{59}$ T. L. Vasconcelos, B. S. Archanjo, B. S. Oliveira, R. Valaski, R. C. Cordeiro, H. G. Medeiros, C. Rabelo, A. Ribeiro, P. Ercius, C. A. Achete, A. Jorio, and L. G. Cançado, Adv. Opt. Mater. 6, 1800528 (2018).

${ }^{60}$ H. Miranda, C. Rabelo, T. L. Vasconcelos, L. G. Cançado, and A. Jorio, Phys. Status Solidi RRL 14, 2000212 (2020).

${ }^{61}$ B. S. Oliveira, B. S. Archanjo, R. Valaski, C. A. Achete, L. G. Cançado, A. Jorio, and T. L. Vasconcelos, J. Chem. Phys. 153, 114201 (2020).

${ }^{62}$ C. D. Tschannen, M. Frimmer, G. Gordeev, T. L. Vasconcelos, L. Shi, T. Pichler, S. Reich, S. Heeg, and L. Novotny, ACS Nano 15, 12249 (2021).

${ }^{63}$ S. Heeg, L. Shi, T. Pichler, and L. Novotny, Carbon 139, 581 (2018).

${ }^{64}$ P. Verma, Chem. Rev. 117, 6447 (2017).

${ }^{65}$ T. Schmid, L. Opilik, C. Blum, and R. Zenobi, Angew. Chem., Int. Ed. 52, 5940 (2013).

${ }^{66}$ X. Wang, S.-C. Huang, S. Hu, S. Yan, and B. Ren, Nat. Rev. Phys. 2, 253 (2020). ${ }^{67}$ L. Shi, P. Rohringer, M. Wanko, A. Rubio, S. Waßerroth, S. Reich, S. Cambré, W. Wenseleers, P. Ayala, and T. Pichler, Phys. Rev. Mater. 1, 075601 (2017).

${ }^{68}$ M. Wanko, S. Cahangirov, L. Shi, P. Rohringer, Z. J. Lapin, L. Novotny, P. Ayala, T. Pichler, and A. Rubio, Phys. Rev. B 94, 195422 (2016).

${ }^{69}$ S. Heeg, L. Shi, L. V. Poulikakos, T. Pichler, and L. Novotny, Nano Lett. 18, 5426 (2018).

${ }^{70}$ A. Jorio, L. G. Cançado, S. Heeg, L. Novotny, and A. Hartschuh, Handbook of Carbon Nanomaterials, World Scientific Series on Carbon Nanoscience Vol. 9-10 (World Scientific, 2017), pp. 175-221.

${ }^{71}$ G. S. Duesberg, I. Loa, M. Burghard, K. Syassen, and S. Roth, Phys. Rev. Lett. 85, 5436 (2000).

${ }^{72}$ C. Thomsen and S. Reich, Top. Appl. Phys. 108, 115 (2007).

${ }^{73}$ X. Yang, C. Lv, Z. Yao, M. Yao, J. Qin, X. Li, L. Shi, M. Du, B. Liu, and C.-X. Shan, Carbon 159, 266 (2020).

${ }^{74}$ Á. Rusznyák, V. Zólyomi, J. Kürti, S. Yang, and M. Kertesz, Phys. Rev. B 72, 155420 (2005).

${ }^{75}$ C. D. Tschannen, G. Gordeev, S. Reich, L. Shi, T. Pichler, M. Frimmer, L. Novotny, and S. Heeg, Nano Lett. 20, 6750 (2020).

${ }^{76}$ A. Jorio, A. G. Souza Filho, G. Dresselhaus, M. S. Dresselhaus, R. Saito, J. H. Hafner, C. M. Lieber, F. M. Matinaga, M. S. S. Dantas, and M. A. Pimenta, Phys. Rev. B 63, 245416 (2001).

${ }^{77}$ N. Bonini, M. Lazzeri, N. Marzari, and F. Mauri, Phys. Rev. Lett. 99, 176802 (2007).

${ }^{78}$ M. Steiner, M. Freitag, V. Perebeinos, J. C. Tsang, J. P. Small, M. Kinoshita, D. Yuan, J. Liu, and P. Avouris, Nat. Nanotechnol. 4, 320 (2009).

${ }^{79}$ R. S. Alencar, C. Rabelo, H. L. S. Miranda, T. L. Vasconcelos, B. S. Oliveira, A. Ribeiro, B. C. Públio, J. Ribeiro-Soares, A. G. S. Filho, L. G. Cançado, and A. Jorio, Nano Lett. 19, 7357 (2019).

${ }^{80}$ G. Xie, D. Ding, and G. Zhang, Adv. Phys.: X 3, 1480417 (2018).

${ }^{81}$ Z. Tian, K. Esfarjani, J. Shiomi, A. S. Henry, and G. Chen, Appl. Phys. Lett. 99, 053122 (2011).

${ }^{82}$ K. Kneipp, Y. Wang, H. Kneipp, I. Itzkan, R. R. Dasari, and M. S. Feld, Phys. Rev. Lett. 76, 2444 (1996).

${ }^{83}$ A. Jorio, M. Kasperczyk, N. Clark, E. Neu, P. Maletinsky, A. Vijayaraghavan, and L. Novotny, Nano Lett. 14, 5687 (2014).

${ }^{84}$ P. Rohringer, L. Shi, P. Ayala, and T. Pichler, Adv. Funct. Mater. 26, 4874 (2016). 